\title{
An Overview on Deep Learning in Image Super- Resolution for Advanced Machine Vision System
}

\begin{abstract}
Meet Kumari ${ }^{1, *}$
${ }^{1}$ Department of ECE, Chandigarh University, Punjab, India

*Corresponding author. Email: meetkumari08@yahoo.in

ABSTRACT

Image spatial resolution means the ability of the sensor to measure the smallest pixel size object. It focuses on recovering a less resolution (LR) image to high resolution (HR) image observations. Due to the exceptional research and application realizations of machine learning at home and abroad, the implementation effect of machine learning algorithms in image super resolution will be enormous. For this, deep learning has become a powerful learning tool for computer vision works. Furthermore, the performance of image super-resolution methods is showing significantly improved by using deep learning. In this paper, the basic image super-resolution methods based on deep learning have been discussed in detail along with the latest applications using super-resolution techniques. In addition, the current open issues and challenges for future research work are discussed. Finally, the main application areas of image superresolution based on deep learning domain are presented.
\end{abstract}

Keywords: Deep learning, Image processing, Machine-learning, Methods, Super-resolution.

\section{INTRODUCTION}

In today's life, image is an important source for users to exchange and obtain the abundant information in the form of photos, graphics, two dimensional (2D) data, three dimensional (3D) data, magnetic resonance (MR) etc. Image processing is used to obtain reliable information in the image. Conventionally, the recent image processing technologies focus on image enhancement, restoration, compression, extraction, transformation and super-resolution in machine vision system. With the evolution of information technology, the super-resolution has made great advancement in image processing. Image super-resolution (SR) is a process which means to the reconstruct high-resolution (HR) from low resolution (LR) images. It is a significant image processing techniques class in image processing and computer vision [1]. It provides lots of applications like surveillance and security, medical imaging, machine vision system etc. Besides, it also improves the image perceptual quality and computer vision tasks. As it is very inherently ill-posed and challenging as there are different HR images regarding a single LR image [2]. With the fast deep learning schemes development in previous years, SR models based on deep learning networks have been actively achieved and explored the conventional performance on different SR benchmarks [3]. Various kinds of deep learning techniques have been utilized to handle SR tasks ranges from previous Convolutional Neural Networks (CNN) to latest SR approaches utilizing Generative Adversarial Nets (GAN). Generally, the SR algorithms family utilizing deep learning techniques differentiate from others in various aspects such as types of network architectures, types of loss functions, types of learning principles and techniques [1, 4]. In last decade, deep convolutional neural networks have been utilized in various research areas because of its image classification achievements. It also shows excellent results on distinct computer vision areas like object detection and face recognition. There are some factors which plays an important role in this progress such as an effective training implementation on powerful GPUs, and proper activation function usage. Most of the advanced SR methods have taken the merits of deep learning approaches. These models help in mapping between HR and LR images and need pre as well as post processing [5]. Although it has wide applications in different areas such as face hallucination, video surveillance, image enhancement, remote sensing, inverse signal estimation and limited low-resolution information problem in spatial resolution, still it is difficult to solve. This motivates for the successful and 
appropriate method for exploring an efficient image spatial resolution.

In this paper, a comprehensive review of latest image super-resolution with deep learning has been presented. However, there are certain existing SR reviews in literature, but this paper mainly focused on latest image SR techniques based on deep-learning, while most of the previous works focused at traditional SR algorithms survey. Contrary to the existing reviews, this review takes definite deep learning based latest advances of image SR methods in a comprehensive and systematic manner. In this paper, Section 2 illustrates the comprehensive literature review. Section 3 and 4 presents the deep learning models for image SR and potential challenges respectively. Section 5 and 6 presents the applications and conclusion respectively.

\section{LITERATURE REVIEW}

The literature review of recent image superresolution methods are as follows:

In [1], a summarized review on latest and advance image super resolution utilizing deep learning methods has been presented. Here, the grouping of existing review methods is done into three categories namely supervised, unsupervised and domain-specific. Also, some essential issues like publicly present benchmark datasets as well as performance evaluation metrics have been covered. It also describes the various future opportunities to be further directed by the community.

In [2], an advanced enhanced deep super-resolution network (EDSR) with higher performance as compared to latest SR methods has been developed. The notable performance enhancement of the proposed model is because of optimization in conventional residual networks by removing unnecessary modules. The performance can be enhanced by model size expanding while stabilize the training method. In addition, a novel multi-scale deep super-resolution system (MDSR) along with training technique to redesign high-resolution different upscaling images' parameters has been proposed. The proposed method shows the excellent performance over the traditional methods on benchmark datasets.

In [3], an accurate single-image SR method has been presented. The proposed method utilised a deep learning convolution network motivated by VGG-net utilized for ImageNet category. It shows that improving the network depth provide a significant enhancement in accuracy. It also performs well as compared to current accuracy methods and visual enhancement.

In [4], a super-resolution generative adversarial network (SRGAN) has been presented. It has the capability of deducing photo-realistic real images for four fold up-scaling parameters. For this a perceptual loss function has been proposed which contains an adversarial lack along with content lack age. It helps in recovering photo-realistic textures on public benchmarks from stiffly down sampled images. It also shows the mean-opinion-score (MOS) largely sufficient gains using SRGAN in rems of perceptual quality.

In [6], a compact hourglass-shape CNN shape for better and faster $\mathrm{Sr}$ has been proposed. It is designed to introduce the deconvolution layer for learning mapping from the low-resolution image at end of network, formulate the mapping layer through input dimension feature shrinking before expanding and mapping back afterwards and to adopt the mapping layers and smaller filter sizes. The proposed model helps in attaining high speed greater than 40 times having superior restoration quality. Also the parameter settings to gain the real-time performance on CPU with improved performance have been presented.

In [7], a sparse coding network model specific designed for SR incarnated as a deep neural network over training data has been demonstrated. It shows that the proposed network enhances the SR performance for incremental and fixed scaling parameters. It can be extended for images' robust handling with degradation like blurring and noise. The conducted subjective assessment has been analysed to investigate different SR methods. It is observed on large range of images and shows that improvement as compared to existing conventional methods for perceptual and quantitative scaling factor.

In [8], the deep Laplacian Pyramid SR network for accurate and fast image SR has been proposed. It shows the progressively reconstruction of high resolution images' the sub-band residuals at various pyramid levels. It directly draw out characteristics from the LR image input space and demands low computational loads. This network has been trained using deep supervision Charbonnier loss functions and attains highquality image recreation. Moreover, to share parameters with in recursive layers and pyramid levels has been utilized. Extensive qualitative as well as quantitative investigation on benchmark datasets presents that the proposed technique performs appropriate against the conventional techniques in the form of image and runtime quality.

In [9], a novel image SR method based on CNN has been proposed to learn the upsampling, feature extraction and HR reconstruction units for end trainable CNN. Here, a combined shallow and deep network has been trained where shallow network having weaker learning capability replace the main image content structure and deep network having strong representation power collect the high frequency information. The propped approach is evaluated on largely adopted data sets as well as compares against conventional methods and provides insights for further research. 
In [10], a deep depth SR method to learn the LR to HR mapping has been proposed. It is hybrid in energy reduction formulation, where DNN learns color depth correlation, depth field statistics and unary term to impose the depth image local structure. It shows the high performance as compared to traditional depth image SR methods.

In [11], an hyperspectral image (HSI) SR approach by joining a spatial constraint (SCT) technique with a deep spectral difference CNN (SDCNN) model has been presented. It helps in resolving HIS produced by reconstructed HR to the input LR HSI, learn the mapping between the LR HSI and HR HSI through experiments for indoor and indoor places. The comparison validates the high performance of proposed method as compared to traditional methods proving spectral information preservation.

Moreover, from the review in [12-18] it is clear that still there is requirement of survey that describes the SR with challenges and applications.

\section{TAXONOMY OF RESEARCH}

The summary of taxonomy of research based on deep learning models for image super resolution is presented in Figure 1.

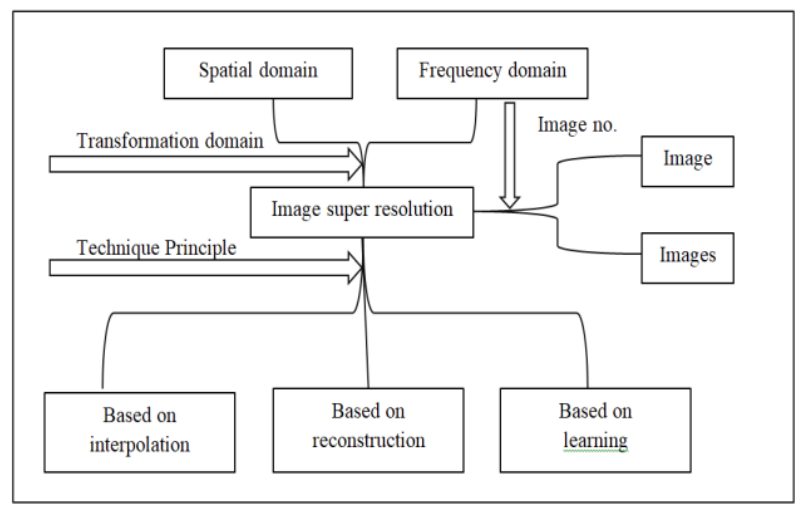

Figure 1 taxonomy of research [19-34]

DNN have been extensively used for lots of applications related to image processing like human pose recovery, image super- resolution, image ranking, image recognition, image privacy protection and image restoration. Here, various advanced DNN for image SR are discussed as follows: [19]

1. SRCNN: This method shows the application in cardiac MR images by simplifying the LR to HR mapping issue by residual learning and permits for deeper training network to obtain enhanced performance as shown in Fig. 1 [20]. In addition, it is data-adaptive and consists of multi-input image extension to attain a good SR image quality. Its quality can be further enhanced by multiple images which are acquired from distinct slice directions and provides the high signal to noise ratio (SNR) [21] The CNN has been evolved fast in the last 20 years. The CNN model to resolve the SISR problem is named as super-resolution convolutional neural network (SRCNN). Here, the goal is observe a suitable model which can be used to find $Y=f(X)$ on not observed examples X. SRCNN includes the paces as follows: [22]

- Pre-processing

- Feature extraction

- Non-linear mapping

- Reconstruction

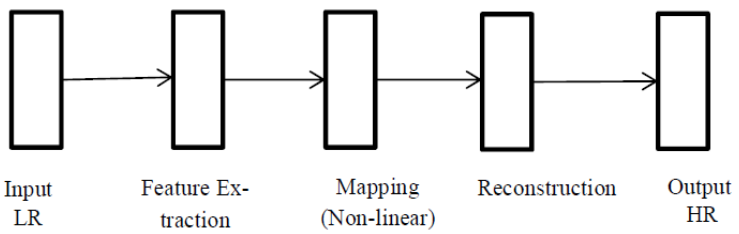

Figure 2 A SRCNN model for SR

2. VDSR and DRCN: Encouraged by the very deep networks success, there are two models viz. very deep convolutional networks (VDSR) and deeply recursive convolutional network (DRCN). VDSR is trained using a learning rate very high to accelerate the speed convergence and explosion problem controlled by whilst gradient clipping [22]. In spite of whole image predicting as processed in SRCNN, residual connection is used to impact the model for training about differentiate between inputs as well output. At borders zeros padding is done to restrict the quickly reducing problem to get advantage from residual learning. In DRRN both local residual and global residual connections are used. Here, global residual learning is utilized in the specification recursive learning and branch in local residual branch as shown in Fig. 2 and 3 [23]

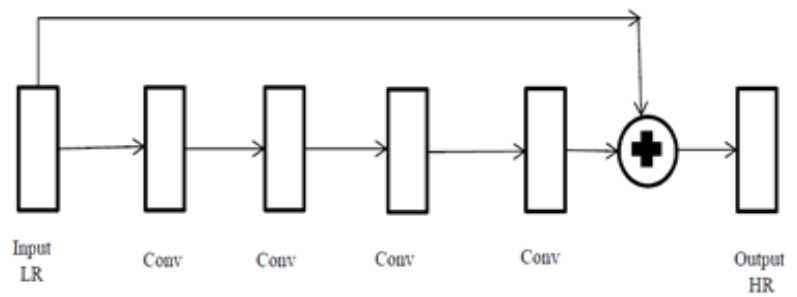

Figure 3 A VDSR model for SR

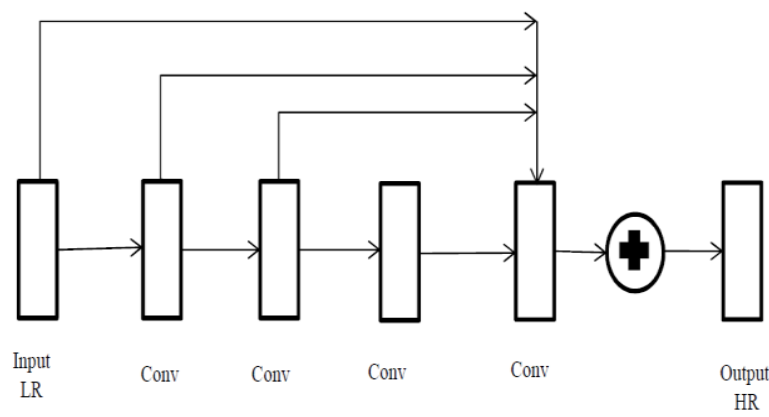

Figure 4 DRCN model for SR 
3. The deep network cascade (DNC) upscale the LR at input side layers to get HR with a small scale factor. The faithful refinements are constructed on observing non-local selfliking to improve the high-frequency patches details. Then the patches are given to layers of a multi-stacked cascading network of collaborative local auto-encoder (CLA) for conformance and noise repression among overlapping patches improved through aforementioned process. It is shown in Fig. [24, 25]

4. Supervised SR: currently, a variety of SR models using deep learning have been proposed, which are focused on supervised learning of deep learning [5]. These are trained with both HR and corresponding LR images. However, the difference is larger between models, but there is set of components combinations like up sampling methods, frameworks, learning strategies and network design. These components can be integrated to generate a hybrid SR model for suitable purposes [26].

\section{SCHEMATIC ANALYIS}

The schematic analysis of the deep learning models for image super resolution is presented in Figure 5.

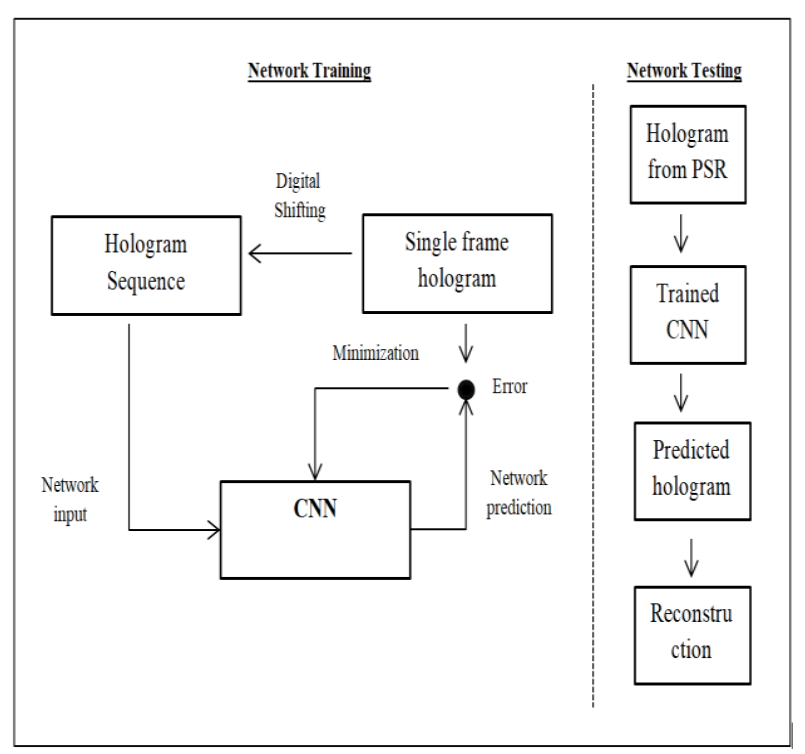

Figure 5 Schematic analysis

The data set of training is generated on the basis of single-frame hologram produced as hologram sequence. Then the image sequence is synthesized using digitally shifting as well as down-sampling this hologram. A neural network trained to achieve a robust SR is utilized to acquired images. For demonstration, a CNN trained on a neural cell hologram to find the hologram of distinct types of samples. This model provides the robustness and effectiveness of the approach [33], [34]. Some primary components used in this are given as follows:
- Pre-upsampling SR: it is a very simple method of mapping LR image to $\mathrm{HR}$ image using conventional upsampling algorithms. It shows the end to end mapping from LR image to HR image. Here, LR images are up-sampled to HR images with suitable size using conventional methods, and then CNN are applied for reconstructing HR images in deep learning [27].

- Post-sampling SR: to enhance the computational capability and using deep learning with full usability to improve SR automatically, post sampling is used. Here, most computation is performed in LR images by restoring the predefined end-to-end layers based up-sampling [28].

- Progressive Up-sampling SR: It is embraced by Laplacian pyramid SR (LapSRN) network. These models are based on cascaded CNN and recreate $\mathrm{HR}$ images. At each level, images are up-sampled to HR an defined by CNN in deep learning [29].

- Iterative Up-and-down Sampling SR: To capture the LRHR mutual dependency in better way, an effective back-projection is used in SR. it is known as Iterative Up-and-down Sampling SR which computes the reconstruction error and fuse back to HR image intensity [21].

\section{DISCUSSIONS}

However, SR methods have been produced for many years, but there are various challenges for image SR. Some of the major challenges are as follows:

- New Trends in Image Restoration and Enhancement (NTIRE) challenge: It is joined with CVPR and consists of various tasks such as colorization, demonising and SR [1].

- Perceptual Image Restoration and Manipulation (PIRM) challenges: It is joined with ECCV and consists of various tasks. As compared to NTIRE, it aims at trade the other SR smartphone [1].

- Registration strategies: The sub-pixel motion areas between the references frames and others frames in simulation can be presented by an attribute mode. Although they have estimated point-wise and block-wise with complex motion condition under most practical. As Optical flow estimation based approaches to find a comparatively correct point's motion field, but is expensive, noise sensitive, illumination variation, large displacements etc. For this advanced registration methods in SR are essential to enhance the accuracy of motion estimation [30].

- Robust fidelity models: however, the accurate registration techniques have been used, motion errors are certain in real. Thus to overcome the impact of inaccurate registration from model 
construction, researchers are working more on $\mathrm{SR}$. The influence of the registration error is primary data fidelity embodied and provides the conformance constraints of the recovered HR image to the investigated LR image. Also, the worst convergence performance restricts the applications [3].

- SR without explicit motion estimation: In current years, SR techniques without explicit motion estimation are becoming famous as it enable to process sequences with a common motion pattern. Computational efficiency is the main concern about SR without explicit motion estimation [3].

- Requirement for simple design model: However, more layers are desirable in deep learning and recent SR model does not include greater than a hundred layers because of the overfitting issue. As the SR models latest model work on pixel level that need lots of parameters as compared to image classification. With increasing layers, the vanishing gradient becoming an issue. This allows for using simple destines with less parameters and minimum computation [15].

- Well to unknown degradation adopting: As various algorithms greatly depend on predetermined premises that LR images are down-sampling as compared to HR images and at large scale they cannot recover SR images because of lack of LR image learnable features. Also, in the presence of noise, the SR image reconstruction accuracy degrades. Thus for this unknown degradation is used for large number of training examples. h. Different assessment criteria requirements: The conventional investigations like L1/L2 loss help to produce images with low disturbance, but they have still significant disagreement in terms of human perception. Thus it is important to enlarge the assessment criteria for specific applications [22].

Besides these various applications are described as follows:

- Face Image SR: It is also known as face hallucination $(\mathrm{FH})$ which helps in face-related tasks. Face images have greater frowned structured knowledge as compared to generic images, thus including facial prior information into FH. One of straightforward approach to compel the produced images to have the same face-related parameters to ground truth. The MTUN and super-FAN introduce FAN to sure the facial landmarks consistency by the end multi-task learning. The FSRNet utilizes facial landmark heat maps along with face parse mapping. The SICNN focuses at reconstructing the real uniqueness embraces a super-identity dropping function and a domain training technique for stabilization [19].

- Hyperspectral Image SR (HIS): As compared to panchromatic images (PANs), HIS contains various bands providing abundant spectral characteristics and helps different vision tasks. Although, because of hardware issues having high quality HSIs is more complex as compared to PANs and have low LR. Therefore, SR is initiated into this area, and researchers incline to join LR HSIs and HR PANs to find HR HSIs [23-27].

- Real-world Image SR: As LR images used for deep learning based SR models training are produced by RGB down sampling images manually. As the real-world cameras capture 14-bit RAW images and work an operational series by camera ISPs (image signal processors) and then generate RGB 8-bit images. This leads to the real RGB images damage. Thus it is important to use the RGB down sampled image directly for SR. This can be solved by SR-RAW, dual CNN etc. approaches [28-34].

- Video enhancement: Using SR methods, the LR video images are converted into HR video images. It can be used in smartphones, computers etc. [35-39].

- Surveillance: SR techniques can be used for security and surveillance monitoring, which is almost impossible for large-scale HR devices. $\mathrm{SR}$ can be used as Walk sequence or a UAV sequence Surveillance [40].

- Medical diagnosis: SR technologies can be used for medical imaging modalities which consist of magnetic resonance imaging (MRI), positron emission tomography (PET) and functional MRI (fMRI) [41].

- Earth observation sensing: For panchromatic image in satellite communication, SR techniques provide the $\mathrm{HR}$ idea to acquire multi-view and multi-temporal images [42-43].

- Astronomical observation: as the Astronomical system stores the images' series for SR. SR helps in enhancing the astronomical images resolution [44].

- Biometric data identification: SR helps in biometric recognition consisting faces resolution enhancement, iris images and fingerprints. SR helps in detection and recognition of biometric images' resolution [4548].

\section{CONCLUSION}

This review paper surveyed key paper in SR using deep learning in machine vision system. Among these, it is noticed that SR using deep learning approaches have attained advanced high performance. In this paper, the important deep learning methods in SR has been highlighted and discussed. Till now, it is seen that a specific SR deep learning method are the most suitable to provide high performance images, it is greatly depend 
on type of application. Means, any SR method which is suitable for medical image processing may not essentially effective for satellite imaging. Then various potential open challenges and application in deep learning based SR imaging has been discussed that encourages for future based imaging. Thus this review paper has improved the concepts of deep learning models applied to SR and that can be utilized as a summarized guide for the beginner in the field in advanced machine vision systems.

\section{REFERENCES}

[1] Z. Wang, J. Chen, and S.C.H. Hoi. Deep Learning for Image Super-resolution: A Survey, In: IEEE Trans. Pattern Anal. Mach. Intell., : p. 1.

[2] B. Lim, K. M. Lee: Enhanced Deep Residual Networks for Single Image Super-Resolution. In: IEEE Conference on Computer Vision and Pattern Recognition Workshops (CVPRW),2017.

[3] J. Kim, J. K. Lee, K. M. Lee: Accurate Image Super-Resolution Using Very Deep Convolutional Networks. In: IEEE Conference on Computer Vision and Pattern Recognition (CVPR), 2016, pp. 1646-1654.

[4] Ledig, C., Theis, L., Husz, F., Caballero, J., Cunningham, A., Acosta, A., Aitken, A., Tejani, A., Totz, J., Wang, Z., Shi, W.: Photo-Realistic Single Image Super-Resolution Using a Generative Adversarial Network. In: IEEE Conference on Computer Vision and Pattern Recognition (CVPR), 2017, pp. 4681-4690.

[5] P. Shamsolmoali, M. Zareapoor: Deep convolution network for surveillance records super-resolution. Multimed. Tools Appl. 78, 23815-23829 (2019).

[6] C. Dong, C. C. Loy, X. Tang: Accelerating the Super-Resolution Convolutional Neural Network. In: Computer Vision -15 ECCV, 2016, p. 391-407

[7] D. Liu, S. Member, Z. Wang, B. Wen, S. Member, J. Yang, W. Han, T. S. Huang: Robust Single Image Super-Resolution via Deep Networks With Sparse Prior. IEEE Trans. Image Process. 25, 2016, pp. 3194-3207.

[8] W. Lai, J. Huang, N. Ahuja, M. Yang: Fast and Accurate Image Super-Resolution with Deep Laplacian Pyramid Networks. In: EEE Conference on Computer Vision and Pattern Recognition (CVPR), 2017, pp. 1-16.

[9] Y. Wang, L. Wang, H. Wang, P. Li: End-to-End Image Super-Resolution via Deep and Shallow Convolutional Networks. IEEE Access. 7, 2019, pp. 31959-31970.

[10]X. Song, Y. Dai, X. Qin: Deep Depth SuperResolution: Learning Depth Super-Resolution using Deep Convolutional Neural Network. In: Computer Vision - ACCV, 2016. pp. 1-13.

[11] Y. Li, J. Hu, X. Zhao, W. Xie, J. Li: Hyperspectral image super-resolution using deep convolutional neural network. Neurocomputing, 2017, pp. 1-39. https://doi.org/10.1016/j.neucom.2017.05.024.

[12] Y. Chen, V. Phonevilay, J. Tao, X. Chen, and R. Xia. The face image super-resolution algorithm based on combined representation learning, Multimed. Tools Appl. (2020a).

[13] J. Choi, J. Kim, M. Cheon, and J. Lee Neurocomputing Deep learning-based image super-resolution considering quantitative and perceptual quality, Neurocomputing. 2020, 398,pp. 347-359.

[14] J. M. Haut, S. Member, R. Fernandez-beltran, M.E. Paoletti, S. Member, J. Plaza, S. Member, and A. Plaza. Remote Sensing Image Superresolution Using Deep Residual Channel Attention, IEEE Trans. Geosci. Remote Sens. 57, 2019, pp. 9277-9289.

[15] M. Kawulok, P. Benecki, S. Member, S. Piechaczek, K. Hrynczenko, D. Kostrzewa, and J. Nalepa. Deep Learning for Multiple-Image SuperResolution, IEEE Geosci. Remote Sens. Lett. 17, 2019, pp. 1062-1066.

[16] P. W. Kim. Image super - resolution model using an improved deep learning - based facial expression analysis, Multimed. Syst. 2020.

[17] K. Li, S. Yang, R. Dong, X. Wang, and J. Huang. Survey of single image super-resolution reconstruction, IET Image Process. 14, 2020, pp. 2273-2290. doi:10.1049/iet-ipr.2019.1438.

[18] X. Li, Y. Wu, W. Zhang, R. Wang, and F. Hou. Deep learning methods in real - time image super - resolution: a survey, J. Real-Time Image Process. 17, pp. 2019, pp.1885-1909.

[19] Q. Chang, K. Hung, J. Jiang: Deep learning based image Super-resolution for nonlinear lens. Neurocomputing, 2017, pp. 1-14. https://doi.org/10.1016/j.neucom.2017.09.035.

[20] S. Network, L. Zhang, P. Wang, C. Shen, L. Liu, W. Wei: Adaptive Importance Learning for Improving Lightweight Image. Int. J. Comput. Vis. 128, 2017, pp. 479-499.

[21] O. Oktay, W. Bai, M. Lee, R. Guerrero, K. Kamnitsas, J. Caballero, A. Marvao, D. S. Cook, D. O. Regan, D. Rueckert: Multi-Input Cardiac Image Super-Resolution using Convolutional Neural Networks.

[22] V. Khanh, H. J. Ren, X. X. Sophia, Z. Gang, V. Masero, A. Hussain: Super-resolution : A Survey. 
Intenational J. Autom. Comput. 16, 2019, pp. 413426.

[23] X. Li, Y. Wu, W. Zhang, R. Wang, F. Hou: Deep learning methods in real - time image super resolution : a survey. J. Real-Time Image Process. 2019. https://doi.org/10.1007/s11554-019-00925-3.

[24] K. Hayat: Multimedia super-resolution via deep learning: A survey. Digit. Signal Process. A Rev. J. 81, 2018, pp. 198-217. https://doi.org/10.1016/j.dsp.2018.07.005.

[25] Zhang, X., Li, C., Meng, Q., Liu, S., Zhang, Y., Wang, J.: Infrared Image Super Resolution by Combining Compressive Sensing and Deep Learning. Sensors. 18, 2018, pp. 2587

[26] Y. Wen, B. Sheng, P. Li, W. Lin, D.D. Feng: Deep Color Guided Coarse-to-Fine Convolutional Network Cascade for Depth Image SuperResolution. IEEE Trans. Image Process. 28, 2018, pp. 994-1006.

[27] Z. He, S. Member, S. Tang, J. Yang, Y. Cao: Cascaded Deep Networks with Multiple Receptive Fields for Infrared Image Super-Resolution. IEEE Trans. Circuits Syst. Video Technol. 29, 2018, pp. 2310-2322

[28] L. Yue, H. Shen, J. Li, Q. Yuan, H. Zhang: Image superresolution : The techniques, applications , and future. Signal Processing. 128, 2016, pp. 389408.

[29] X. Zhao, Y. Zhang, T. Zhang, X. Zou: Channel Splitting Network for Single MR Image. IEEE Trans. Image Process. 28, 2019, pp. 5649-5662.

[30] A. S. Review, Deep Learning Algorithms for Single Image Super-Resolution: A Systematic Review, Electronics. 10, 2021, pp. 867.

[31] Y. L.Wang, H. Wang, and P. Li. End-to-End Image Super-Resolution via Deep and Shallow Convolutional Networks, IEEE Access. 7, 2019, pp. 31959-31970.

[32] W. Yan, X. Zhang, Y. Tian, W. Wang, J. Xue, and Q. Liao. Deep Learning for Single Image SuperResolution: A Brief Review, IEEE Trans. Multimed. 12, 2019, pp. 3106-3121. doi:10.1109/TMM.2019.2919431.

[33] H. Liu, J. Han, S. Hou, L. Shao, and Y. Ruan. Single Image Super-Resolution Using a Deep Encoder-Decoder Symmetrical Network with Iterative Back Projection, Neurocomputing, 282, 2018, pp. 52-59. doi:10.1016/j.neucom.2017.12.014.

[34] Z.H.L. Uo, A.B.Y. Urt, R.I.S. Tahl, A. Ndy, L. Ambrechts, V.E.R. Eumers, D.R.B. Raeken, and L. Iesbet. Pixel super-resolution for lens-free holographic microscopy using deep learning neural networks, Opt. Express, 27, 2019, pp. $13581-13595$

[35] Arun, M., E. Baraneetharan, A. Kanchana, and S. Prabu. "Detection and monitoring of the asymptotic COVID-19 patients using IoT devices and sensors." International Journal of Pervasive Computing and Communications (2020).

[36] Kumar, M. Keerthi, B. D. Parameshachari, S. Prabu, and Silvia liberata Ullo. "Comparative Analysis to Identify Efficient Technique for Interfacing BCI System." In IOP Conference Series: Materials Science and Engineering, vol. 925, no. 1, p. 012062. IOP Publishing, 2020.

[37] L. Zhen, Y. Zhang, K. Yu, N. Kumar, A. Barnawi and Y. Xie, "Early Collision Detection for Massive Random Access in Satellite-Based Internet of Things," IEEE Transactions on Vehicular Technology, vol. 70, no. 5, pp. 5184-5189, May 2021, doi: 10.1109/TVT.2021.3076015.

[38] L. Tan, K. Yu, A. K. Bashir, X. Cheng, F. Ming, L. Zhao, X. Zhou, "Towards Real-time and Efficient Cardiovascular Monitoring for COVID-19 Patients by 5G-Enabled Wearable Medical Devices: A Deep Learning Approach", Neural Computing and Applications, https://doi.org/10.1007/s00521-021-06219-9.

[39] L. Tan, K. Yu, F. Ming, X. Cheng, G. Srivastava, "Secure and Resilient Artificial Intelligence of Things: a HoneyNet Approach for Threat Detection and Situational Awareness", IEEE Consumer Electronics Magazine, 2021, doi: 10.1109/MCE.2021.3081874.

[40] Puttamadappa, C., and B. D. Parameshachari. "Demand side management of small scale loads in a smart grid using glow-worm swarm optimization technique." Microprocessors and Microsystems 71 (2019): 102886.

[41] Parameshachari, B. D., H. T. Panduranga, and Silvia liberata Ullo. "Analysis and computation of encryption technique to enhance security of medical images." In IOP Conference Series: Materials Science and Engineering, vol. 925, no. 1, p. 012028. IOP Publishing, 2020.

[42] Pham, Dung V., Giang L. Nguyen, Tu N. Nguyen, Canh V. Pham, and Anh V. Nguyen. "Multi-topic misinformation blocking with budget constraint on online social networks." IEEE Access 8 (2020): 78879-78889.

[43] Naeem, Muhammad Ali, Tu N. Nguyen, Rashid Ali, Korhan Cengiz, Yahui Meng, and Tahir Khurshaid. "Hybrid Cache Management in IoTbased Named Data Networking." IEEE Internet of Things Journal (2021).

[44] K. Yu, M. Arifuzzaman, Z. Wen, D. Zhang and T. Sato, "A Key Management Scheme for Secure 
Communications of Information Centric Advanced Metering Infrastructure in Smart Grid," IEEE Transactions on Instrumentation and Measurement, vol. 64, no. 8, pp. 2072-2085, August 2015.

[45] K. Yu, L. Tan, X. Shang, J. Huang, G. Srivastava and P. Chatterjee, "Efficient and PrivacyPreserving Medical Research Support Platform Against COVID-19: A Blockchain-Based Approach”, IEEE Consumer Electronics Magazine, doi: 10.1109/MCE.2020.3035520.

[46] Z. Guo, Y. Shen, A. K. Bashir, M. Imran, N. Kumar, D. Zhang and K. Yu, "Robust Spammer Detection Using Collaborative Neural Network in Internet of Thing Applications", IEEE Internet of Things Journal, vol. 8, no. 12, pp. 9549-9558, 15 June15, 2021, doi: 10.1109/JIOT.2020.3003802.

[47] Le, Ngoc Tuyen, Jing-Wein Wang, Duc Huy Le, Chih-Chiang Wang, and Tu N. Nguyen. "Fingerprint enhancement based on tensor of wavelet subbands for classification." IEEE Access 8 (2020): 6602-6615.

[48] Rajendran, Ganesh B., Uma M. Kumarasamy, Chiara Zarro, Parameshachari B. Divakarachari, and Silvia L. Ullo. "Land-use and land-cover classification using a human group-based particle swarm optimization algorithm with an LSTM Classifier on hybrid pre-processing remote-sensing images." Remote Sensing 12, no. 24 (2020): 4135. 